\title{
Dependency on mask ventilation after acute respiratory failure in the intermediate care unit
}

\author{
A. Cuvelier*,, C. Viacroze*, J. Bénichou\#, L.C. Molano*, M-F. Hellot", \\ D. Benhamou* and J-F. Muir*'
}

ABSTRACT: The predictive factors for long-term dependency (LTD) on noninvasive ventilation (NIV) immediately after acute hypercapnic respiratory failure (AHRF) have not been identified.

The present authors studied 42 patients with chronic obstructive pulmonary disease (COPD) and 58 non-COPD patients successfully treated by NIV for an AHRF episode. Parameters at stable state, at admission for AHRF and during a 1-yr follow-up were compared in patients with or without LTD-NIV at discharge.

The incidence of LTD-NIV was $39 \%$ in non-COPD patients and 19\% in COPD patients. Based on multivariate analysis with stepwise logistic regression, lower baseline $\mathrm{pH}$ values and noninfectious causes of AHRF were independently associated with LTD-NIV. Mutually adjusted odds ratios were found to be $1.316(95 \%$ confidence interval $(\mathrm{Cl})=1.127-1.536)$ for a 0.01 decrease of baseline $\mathrm{pH}$ value and $5.1(95 \% \mathrm{Cl}=1.8-14.0)$ for a noninfectious cause of AHRF. Outcome after $1 \mathrm{yr}$ was poor in COPD patients.

Long-term dependency on noninvasive ventilation is not an uncommon situation after resolution of an acute hypercapnic respiratory failure episode, especially in patients with non-chronic obstructive pulmonary disease causes of respiratory failure. The present study raises the need for prospective validation of a weaning protocol in patients managed by noninvasive ventilation for an acute hypercapnic respiratory failure episode.

KEYWORDS: Acute respiratory failure, chronic obstructive pulmonary disease, chronic respiratory failure, noninvasive ventilation, weaning

oninvasive ventilation (NIV) is a cornerstone in the management of patients with acute hypercapnic respiratory failure (AHRF) [1, 2]. Several studies have also reported the clear beneficial effects of domiciliary NIV in large series of patients with chronic hypercapnic respiratory failure during the course of restrictive pulmonary diseases [3-5]. However, the benefits of domiciliary NIV remain controversial in patients with chronic respiratory failure and chronic obstructive pulmonary disease (COPD) [6-13].

Long-term domiciliary NIV is usually initiated outside the context of an AHRF episode in patients with chronic respiratory failure, symptomatic nocturnal alveolar hypoventilation, frequent hospitalisations and progressive worsening clinical status and arterial blood gases (ABGs) $[8,9,14]$. However, domiciliary NIV is also initiated in intensive care units (ICUs) after an AHRF episode with impossible or difficult weaning, but criteria to determine who should benefit from long-term domiciliary NIV after this episode have not been clearly established and indications vary between medical teams. Most prospective studies concerning NIV in acute care do not examine this issue, as they focus on the short-term success/ failure of NIV [1, 15-17]. They do not assess followup after discharge and rarely provide information about the readmission rates for recurrent AHRF or on the incidence of domiciliary ventilatory assistance at discharge [14].

The aim of the present study was to establish the predictive factors for long-term dependency (LTD)-NIV following the resolution of an AHRF episode in patients managed in a respiratory intermediate care unit (RICU). Parameters at stable state (during the 6 months prior to the AHRF episode) and at admission into the RICU for the AHRF episode were compared in patients with and without LTD-NIV at discharge. Finally, the present authors compared outcome in patients with and without LTD-NIV during a 1-yr follow-up after discharge from the RICU.

\section{PATIENTS AND METHODS \\ Patients}

During two consecutive years in the RICU of the present authors, all patients admitted for an
AFFILIATIONS

*Service de Pneumologie,

\#Unité de Biostatistique, and

"UPRES EA 3830, IFR MP23, Hôpital

de Bois-Guillaume and Hôpital

Charles Nicolle, Centre Hospitalier

Universitaire de Rouen, Rouen,

France.

CORRESPONDENCE

A. Cuvelier

Service de Pneumologie et Unité de

Soins Intensifs Respiratoires

Hôpital de Bois-Guillaume

Centre Hospitalier Universitaire de

Rouen

76031 Rouen-Cedex

France

Fax: 33232889094

E-mail: antoine.cuvelier@chu-

rouen.fr

Received:

October 112004

Accepted after revision:

May 032005 
AHRF episode, who were successfully managed by NIV and subsequently discharged home with or without domiciliary NIV, were retrospectively assessed. AHRF was defined as an exacerbation of dyspnoea lasting for $<1$ week associated with respiratory acidosis defined by $\mathrm{pH} \leqslant 7.35$ and carbon dioxide arterial tension $\left(\mathrm{Pa}_{\mathrm{a}} \mathrm{CO}_{2}\right) \geqslant 6.6 \mathrm{kPa}$. Patients who required invasive ventilation during their stay in the RICU were not included in the present study in order to obtain a homogeneous sample of patients successfully treated by NIV alone. All the patients included were required to be free from previous long-term ventilation and were categorised into COPD and non-COPD subgroups according to past medical history and lung function tests. The following patients were excluded from the study: 1) those in whom long-term NIV was considered to be unethical because of the lack of potential benefit; 2) patients in whom long-term NIV was impossible to perform under satisfactory technical conditions; and 3) patients who refused domiciliary NIV.

The weaning process in the RICU of the current authors is standardised as follows. When the patient's condition has stabilised and the triggering factor of AHRF has been controlled, the length of NIV is gradually reduced, during the daytime period only, under close monitoring of clinical respiratory status, and ABG drawn in the early morning and at the end of the day. When daytime NIV is stopped, the possibility of stopping nocturnal NIV is then assessed, on the basis of the patient's clinical condition and ABG values at the end of the following day. Repeat ABGs are performed at any time as required depending on clinical status. Weaning is considered successful when neither clinical alteration nor respiratory acidosis $(\mathrm{pH} \leqslant 7.35)$ is observed within eight consecutive days after completely discontinuing the NIV. If weaning fails, a further attempt is performed using the same procedure. All decisions concerning the initiation of domiciliary long-term NIV were made by three physicians after discussion with the patient, the patient's relatives and nursing staff.

Based on this practice, the present authors define LTD-NIV immediately following AHRF when all of the three following criteria are present: 1) stable resolution of the triggering factor; 2) inability to stop NIV for at least eight consecutive days (after two attempts) because of worsening clinical status, a rise in $\mathrm{Pa}_{1} \mathrm{CO}_{2}$ with respiratory acidosis $(\mathrm{pH} \leqslant 7.35)$ and/or recurrent AHRF without any identifiable cause; and 3) discharge home with domiciliary NIV.

In some cases, long-term NIV is considered as unethical because of the lack of potential benefits, the impossibility to perform long-term NIV under satisfactory technical or safety conditions, or because a patient refuses domiciliary NIV. Patients discharged home with NIV are managed with the technical assistance of a home care network and assessed at outpatient clinics every 3 months.

\section{Management in RICU}

The present authors' RICU is a 10-bedded unit with an annual admission rate of $\sim 350$ patients, $>75 \%$ of whom present with AHRF. All of the nurses and respiratory therapists are specifically trained in NIV and a senior respiratory physician dedicated to the unit is on call $24 \mathrm{~h}$.
NIV is administrated in the RICU on the basis of standardised protocols. As a first line approach, all patients are ventilated with a face mask and in positive pressure support mode. The initial choice of ventilator is left to the discretion of the duty physician and is influenced primarily by patient tolerability, success/failure of a previous NIV test and the nature of the respiratory impairment at baseline. Inspiratory positive airway pressure is set at a maximal value depending on the patient comfort and ABGs. Expiratory positive pressure is set at a minimal value except in patients with COPD and in those with obstructive sleep apnoea. All patients are ventilated with a back-up frequency rate of $12 \cdot \mathrm{min}^{-1}$. Inspiratory oxygen fraction is set in order to obtain an arterial oxygen saturation $\geqslant 92 \%$. The inspiratory/expiratory ratio is set depending on the cause of the respiratory impairment (lower in COPD patients and higher in those with restrictive disorders). Changes to the ventilatory settings are decided by the duty physician. After admission with AHRF, patients are ventilated, allowing short periods of spontaneous breathing to permit eating, drinking, performing of bronchial drainage, administering of nebulised bronchodilators and for washing. Clinical state and ABGs are monitored according to an international consensus statement [18]. The intubation criteria are adapted from BROCHARD et al. [1] requiring one major or two minor criteria to indicate endotracheal intubation. The major criteria include respiratory arrest, gasping, coma, agitation requiring sedation, heart rate $<50$ beats $\cdot \min ^{-1}$ with unconsciousness and unstable haemodynamic status with systolic arterial pressure $<70 \mathrm{mmHg}$. The minor criteria include respiratory rate $>35$ breaths $\cdot \mathrm{min}^{-1}$ and above the rate at admission, arterial oxygen tension $<6 \mathrm{kPa}$ despite oxygen therapy and deterioration of the encephalopathy score.

The cause of the episode of AHRF was established after careful review of each patient's clinical records. The diagnosis of pneumonia was made in the presence of lung infiltrates on chest radiographs, combined with any three of the following factors: fever, positive blood cultures, leukocytosis or potential pathogenic bacterial cultures from sputum, bronchial aspiration or transtracheal aspirates. The current authors defined bronchial infection as the presence of purulent sputum, associated with an increase in baseline dyspnoea and in sputum volume.

\section{Statistical analysis}

Continuous variables are presented as mean $\pm \mathrm{SD}$, unless otherwise indicated. A univariate analysis was performed in order to determine the individual predictive clinical and functional characteristics for LTD-NIV when collected at steady state and at admission to the RICU for the AHRF episode. In particular, the associations between each discontinuous variable and LTD-NIV were assessed by the Pearson Chi-squared test or Fisher's exact test, as appropriate. The corresponding associations with continuous variables were assessed by the Mann-Whitney U-test. In order to correct for multiple testing, the Bonferroni correction was applied. Specifically, $\mathrm{p}$-values $<0.05 / 24$ or 0.0021 were considered as significant, since 24 tests were performed. Variables that appeared to be independently associated with LTD-NIV at a probability of $10 \%$ were used in the multivariate analysis. Forward stepwise logistic regression was used to determine 
variables that were independently associated with LTD-NIV. Changes in $\mathrm{pH}$ and $\mathrm{Pa}, \mathrm{CO}_{2}$ during the stay in the RICU between patients with or without subsequent LTD-NIV were performed using a two-way ANOVA. Day-by-day comparisons were performed using the Mann-Whitney U-test. In patients who stopped NIV within 7 days, the last available value was carried forward (last observation carried forward analysis).

\section{RESULTS}

\section{Patient characteristics}

A total of 106 patients met the inclusion criteria for the study. Domiciliary NIV on discharge was not considered in six patients who were invalid and bedridden $(n=2)$ or who refused home NIV $(n=4)$. These six patients were older (mean age $76.3 \pm 9.3 \mathrm{yrs}$ ) than the other patients included in the study. Four of these patients had COPD. The median baseline $\mathrm{pH}$ in these patients was 7.39 (range 7.34-7.43) and median $\mathrm{Pa}_{2} \mathrm{CO}_{2}$ was $7.0 \mathrm{kPa}$ (range $5-9.8 \mathrm{kPa}$ ). A total of four patients (two with COPD and two without COPD) died during the 1-yr follow-up period.

The remaining 100 patients (42 with COPD and 58 without COPD) were included in the analysis. The respiratory conditions present in the non-COPD patients were as follows: chest wall deformities $(n=21)$; obesity defined as body mass index (BMI) $>30 \mathrm{~kg} \cdot \mathrm{m}^{-2}(\mathrm{n}=19)$; complications of tuberculosis $(\mathrm{n}=10)$; primary bronchiectasis $(\mathrm{n}=6)$; neuromuscular disease $(n=2)$. The triggering factor for AHRF was identified in 93 patients as the following: bronchial infection $(n=53)$; infectious pneumonia $(n=14)$; progression of underlying chronic respiratory failure $(n=13)$; acute pulmonary oedema $(n=6)$; excess sedative use $(n=3)$; pneumothorax $(n=2)$; pulmonary embolism $(n=1)$; and episode of aspiration $(n=1)$. No cause was found in seven patients.

\section{LTD-NIV depending on steady state clinical and functional characteristics}

A total of 31 patients (31\%) had LTD-NIV after successful treatment of AHRF. Long-term oxygen therapy had been used previously in 26 patients without LTD-NIV (38\%) and in 18 patients with LTD-NIV $(58 \%$; $p=0.058)$. Fewer of the COPD patients were equipped with home NIV than the non-COPD patients, but the difference was not statistically significant (19\% versus $40 \%$; $\mathrm{p}=0.0279)$. LTD-NIV was not associated with sex, BMI or smoking status (table 1). The incidence of steady state comorbidities, mostly cardiovascular in origin, was similar in patients with or without LTD-NIV.

The proportion of patients hospitalised for AHRF during the previous 3 yrs was similar in non-COPD patients with or without subsequent LTD-NIV (39\% versus $40 \%$; $=0.95$ ). In contrast, most COPD patients with subsequent LTD-NIV had been admitted to hospital during the previous 3 yrs for AHRF ( $75 \%$ versus $41 \%$; $=0.12$ ). There was no significant difference in the number of previous hospital admissions between patients with or without subsequent LTD-NIV with $1.9 \pm 1.6$ and 2.6 \pm 2.1 admissions, respectively. These were similar in the COPD and non-COPD patients.

Steady state lung function tests were not predictive for subsequent LTD-NIV either in the whole patient group or in the COPD or non-COPD subgroups (table 2). LTD-NIV was significantly associated with lower $\mathrm{pH}$ and higher $\mathrm{Pa}_{\mathrm{a}} \mathrm{CO}_{2}$ values at baseline in the whole patient group and in the nonCOPD subgroup (table 3 ).

\section{LTD-NIV depending on characteristics of the episode of AHRF}

The simplified acute physiology score (SAPS) I severity index on admission was not predictive of LTD-NIV in the entire sample or in either patient group. LTD-NIV was less frequent in patients admitted for AHRF of infectious origin, either bronchial infection or pneumonia (fig. 1). Blood neutrophil count was higher on admission in patients without LTD-NIV $\left(10.8 \pm 7.8 \times 1000 \cdot \mathrm{mm}^{-3}\right.$ versus $8.6 \pm 7.7 \times 1000 \cdot \mathrm{mm}^{-3}$ in patients with LTD-NIV), but the difference was not significant $(p=0.009)$. There was no difference in the severity of $\mathrm{pH}$ on admission to the RICU in patients with or without subsequent LTD-NIV with $7.30 \pm 0.03$ versus $7.30 \pm 0.04$, respectively $(p=0.37)$. Similarly, there was no difference in the severity of

TABLE 1 Long-term dependency on noninvasive ventilation (LTD-NIV) following hospitalisation in the authors' respiratory intermediate care unit for an episode of acute hypercapnic respiratory failure depending on characteristics at stable state during the 6 months prior to the episode

\begin{tabular}{|c|c|c|c|c|c|c|c|c|c|}
\hline & \multicolumn{3}{|c|}{ All patients } & \multicolumn{3}{|c|}{ COPD patients } & \multicolumn{3}{|c|}{ Non-COPD patients } \\
\hline Male:female & $43: 26$ & $18: 13$ & 0.69 & $27: 7$ & $5: 3$ & 0.37 & $16: 19$ & $13: 10$ & 0.42 \\
\hline Age yrs & $71.4 \pm 10.2$ & $65.0 \pm 11.6$ & 0.02 & $69.1 \pm 8.8$ & $68.3 \pm 7.8$ & 0.96 & $73.6 \pm 11$ & $63.8 \pm 12.6$ & 0.006 \\
\hline BMI $\mathbf{k g} \cdot \mathrm{m}^{-2}$ & $28.6 \pm 8.9$ & $32.5 \pm 13.3$ & 0.41 & $27.5 \pm 10.5$ & $29.7 \pm 9.7$ & 0.72 & $29.8 \pm 10.6$ & $33.6 \pm 14.5$ & 0.50 \\
\hline ex-smoker & $34(49)$ & $12(39)$ & & $20(59)$ & $5(62)$ & & $14(40)$ & $7(30)$ & \\
\hline nonsmoker & $21(30)$ & $15(49)$ & & $7(21)$ & $2(25)$ & & $14(40)$ & $13(57)$ & \\
\hline active smoker & $14(20)$ & $4(13)$ & & $7(21)$ & $1(13)$ & & $7(20)$ & $3(13)$ & \\
\hline Pack-yrs & $38.9 \pm 10.7$ & $38.6 \pm 14.1$ & 0.54 & $38.7 \pm 25.2$ & $47.5 \pm 18.5$ & 0.23 & $39.2 \pm 26.4$ & $31.9 \pm 15.6$ & 0.68 \\
\hline
\end{tabular}

Data are presented as mean \pm SD or $n(\%)$, unless otherwise indicated. COPD: chronic obstructive pulmonary disease; BMl: body mass index. 


\begin{tabular}{|c|c|c|c|c|c|c|c|c|c|}
\hline \multirow[t]{3}{*}{ TABLE 2} & \multicolumn{9}{|c|}{$\begin{array}{l}\text { Long-term dependency on noninvasive ventilation (LTD-NIV) following hospitalisation in the respiratory intermediate care } \\
\text { unit for an episode of acute hypercapnic respiratory failure depending on lung function tests at stable state during the } \\
6 \text { months prior to the episode }\end{array}$} \\
\hline & \multicolumn{3}{|c|}{ All patients } & \multicolumn{3}{|c|}{ COPD patients } & \multicolumn{3}{|c|}{ Non-COPD patients } \\
\hline & No LTD-NIV & LTD-NIV & $p$-value & No LTD-NIV & LTD-NIV & $p$-value & No LTD-NIV & LTD-NIV & p-value \\
\hline 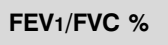 & $60.6 \pm 16.0$ & $66.6 \pm 16.3$ & 0.08 & $51.5 \pm 13.3$ & $52.1 \pm 14.7$ & 0.95 & $70.3 \pm 12.6$ & $72.7 \pm 12.9$ & 0.35 \\
\hline SVC $\%$ pred & $56.5 \pm 18.7$ & $54.3 \pm 17.1$ & 0.74 & $61.7 \pm 20.5$ & $64.7 \pm 19.7$ & 0.54 & $50.4 \pm 14.6$ & $50.5 \pm 14.8$ & 0.95 \\
\hline FVC \% pred & $50.7 \pm 15.8$ & $49.7 \pm 15.0$ & 0.87 & $53.5 \pm 16.6$ & $54.5 \pm 20.0$ & 0.85 & $47.7 \pm 14.6$ & $47.7 \pm 12.5$ & 0.96 \\
\hline TLC \% pred & $75.6 \pm 25.9$ & $63.1 \pm 19.2$ & 0.08 & $91.2 \pm 23.1$ & $83.4 \pm 12.1$ & 0.58 & $57.4 \pm 15.0$ & $55.2 \pm 15.2$ & 0.63 \\
\hline RV \% pred & $104.1 \pm 52.5$ & $85.0 \pm 41.2$ & 0.11 & $134.5 \pm 51.4$ & $121.9 \pm 55.2$ & 0.36 & $71.1 \pm 28.5$ & $69.8 \pm 21.4$ & 0.95 \\
\hline
\end{tabular}

Data are presented as mean \pm SD or $\mathrm{n}$. COPD: chronic obstructive pulmonary disease; FEV1: forced expiratory volume in one second; \% pred: percentage of the predicted value; FVC: forced vital capacity; SVC: slow vital capacity; TLC: total lung capacity; RV: residual volume.

TABLE 3

Long-term dependency on noninvasive ventilation (LTD-NIV) following hospitalisation in the respiratory intermediate care unit for an episode of acute hypercapnic respiratory failure depending on the arterial blood gases at stable state during the 6 months prior to the episode

\begin{tabular}{|c|c|c|c|c|c|c|c|c|c|}
\hline & \multicolumn{3}{|c|}{ All patients } & \multicolumn{3}{|c|}{ COPD patients } & \multicolumn{3}{|c|}{ Non-COPD patients } \\
\hline & No LTD-NIV & LTD-NIV & $p$-value & No LTD-NIV & LTD-NIV & $\mathrm{p}$-value & No LTD-NIV & LTD-NIV & p-value \\
\hline Subjects $n$ & 69 & 31 & & 34 & 8 & & 35 & 23 & \\
\hline $\mathrm{Pa}, \mathrm{O}_{2} / \mathrm{Fl}_{1} \mathrm{O}_{2}$ & $241.2 \pm 131.6$ & $208.2 \pm 112.0$ & 0.08 & $303.0 \pm 97.3$ & $245.8 \pm 114.1$ & 0.19 & $298.8 \pm 60.8$ & $255.5 \pm 55.2$ & 0.03 \\
\hline $\mathrm{pH}$ & $7.42 \pm 0.04$ & $7.38 \pm 0.03$ & 0.0001 & $7.41 \pm 0.04$ & $7.39 \pm 0.03$ & 0.16 & $7.43 \pm 0.04$ & $7.38 \pm 0.03$ & 0.0001 \\
\hline
\end{tabular}

Data are presented as mean $\pm \mathrm{SD}$ or $\mathrm{n}$. COPD: chronic obstructive pulmonary disease; $\mathrm{Pa}_{\mathrm{a}} \mathrm{O}_{2}$ : arterial oxygen tension; $\mathrm{Fl}, \mathrm{O}_{2}$ : inspired oxygen fraction; $\mathrm{Pa}, \mathrm{CO}_{2}$ : carbon dioxide arterial tension; $\mathrm{HCO}_{3}$ : hydrogen carbonate.

hypercapnia on admission in patients with or without subsequent LTD-NIV with $9.6 \pm 1.3$ versus $9.3 \pm 1.2 \mathrm{kPa}$, respectively $(p=0.25)$.

\section{Independent predictive factors of LTD-NIV}

Based on a multivariate analysis with stepwise logistic regression, lower baseline $\mathrm{pH}$ values and a noninfectious cause for the AHRF emerged as the only variables that were independently associated with subsequent LTD-NIV in the whole population of patients. The mutually adjusted odds ratios (ORs) were estimated to be 1.316 (95\% confidence interval (CI) 1.127-1.536; $\mathrm{p}=0.0005$ ) for a 0.01 fall in baseline $\mathrm{pH}$ and $5.1(95 \% \mathrm{CI}=1.8-14.0 ; \mathrm{p}=0.0018)$ for a noninfectious cause of the AHRF. In the whole sample of patients, a baseline $\mathrm{pH}$ value $\leqslant 7.38$ was associated with an OR of $7.3(95 \%$ $\mathrm{CI}=2.5-21.4 ; \mathrm{p}=0.0124)$ for LTD-NIV on discharge, 11.3 $(95 \% \mathrm{CI}=2.6-9.1 ; \mathrm{p}=0.0013)$ in non-COPD patients and 4.1 (95\% CI $=0.7-24.2 ; \mathrm{p}=0.1231)$ in COPD patients.

\section{LTD-NIV depending on immediate outcome in RICU}

Measured $\mathrm{pH}$ values on spontaneous breathing $24 \mathrm{~h}$ after admission to the RICU in patients without subsequent LTDNIV improved significantly (from $7.30 \pm 0.04$ to $7.39 \pm 0.05$; $\mathrm{p}<0.0001)$ but did not improve in patients with subsequent LTD-NIV (from $7.30 \pm 0.03$ to $7.32 \pm 0.04 ; p=0.74$; fig. 2). Measured $\mathrm{Pa}, \mathrm{CO}_{2}$ on spontaneous breathing improved significantly $24 \mathrm{~h}$ after admission to the RICU in patients without LTD-NIV (from $9.3 \pm 1.2$ to $7.4 \pm 1.2 \mathrm{kPa}$; $\mathrm{p}=0.0001$ ), but not in patients with LTD-NIV (from $9.6 \pm 1.3$ to $9.0 \pm 1.3 \mathrm{kPa}$; $\mathrm{p}=0.41$; fig. 2). Similar results were found $48 \mathrm{~h}$ after admission.

Daily changes in ABGs measured on mechanical ventilation were different depending on the presence or absence of subsequent LTD-NIV (figs 3 and 4). However, this difference only appeared after the third day of NIV and was only statistically significant in the non-COPD subgroup.

The length of daily NIV was similar during the first 7 days in patients with or without subsequent LTD-NIV. During the first day, mean NIV use was $16.2 \pm 1.4 \mathrm{~h}$ in patients without LTDNIV and $15.8 \pm 1.6 \mathrm{~h}$ in patients with LTD-NIV. On the second day, mean NIV use was $12.1 \pm 1.9 \mathrm{~h}$ in patients without LTDNIV and $12.4 \pm 2.3 \mathrm{~h}$ in patients with LTD-NIV. Mean daily use fell during the study period until $9.5 \pm 1.9 \mathrm{~h}$ (range $7-18$ ) on discharge in patients with LTD-NIV. The length of NIV in RICU in patients with LTD-NIV was $8.3 \pm 6.8$ days. The 


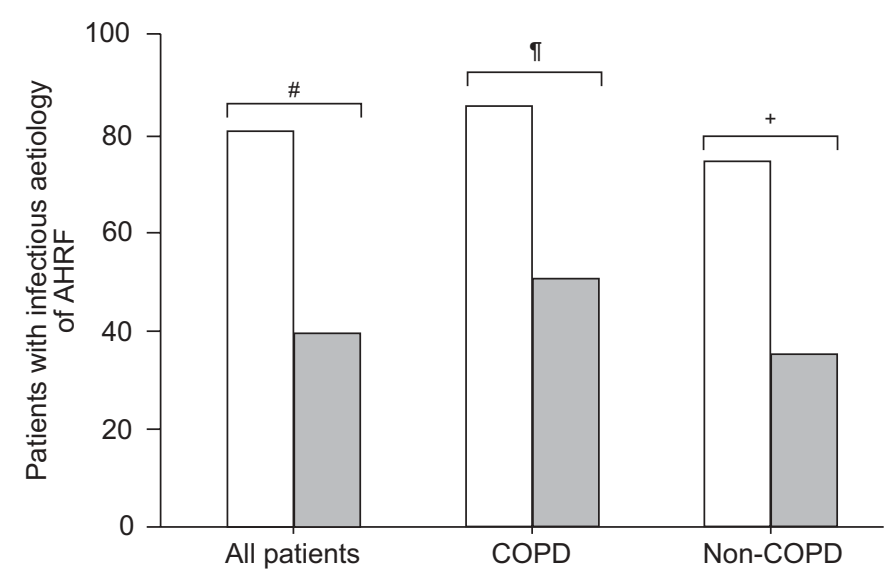

FIGURE 1. Long-term dependency on noninvasive ventilation (LTD-NIV) after hospitalisation in the respiratory intermediate care unit depending on the infectious or noninfectious cause of the episode of acute hypercapnic respiratory failure (AHRF). Data is presented for all patients $(n=100)$, and those with $(n=42)$ and without $(n=58)$ chronic obstructive pulmonary disease (COPD) having ( $(\square)$ or not having ( $\square$ ) LTD-NIV. Patients with AHRF of infectious origin were significantly less likely to be discharged with domiciliary NIV. All comparisons were performed with Pearson Chi-squared test. \#: $p<0.0001 ;{ }^{\uparrow}: p=0.0499 ;{ }^{+}: p<0.0028$

mean stay in the RICU was longer in patients with LTDNIV (14.2 \pm 8.2 versus $10.9 \pm 7.8$ days; $p=0.04)$ although mean hospital stay was similar in patients with or without LTD-NIV $(19.0 \pm 13.8$ versus $18.5 \pm 9.4$ days, respectively; $\mathrm{p}=0.63) . \mathrm{Pa}_{\mathrm{a}} \mathrm{CO}_{2}$ on discharge was higher in patients with LTD-NIV $(7.0 \pm 1.1$ versus $6.2 \pm 0.9 \mathrm{kPa}$, respectively; $\mathrm{p}=0.0005)$.

\section{Clinical follow-up after the episode of AHRF}

All patients were followed up for $\geqslant 1 \mathrm{yr}$ post-discharge. During the follow-up period domiciliary NIV was started in 11 patients because of progressive, deteriorating clinical condition and/or recurrence of AHRF. Treatment, however, was finally stopped in five because of poor tolerance and/or insufficient clinical benefit (table 4). The episode of AHRF on inclusion was the first episode of AHRF in eight of these patients.

No patient had to be tracheostomised during the 1-yr followup. The present authors decided to discontinue domiciliary ventilation in one patient with LTD-NIV in the non-COPD subgroup, because of sustained improvement in clinical parameters and ABGs. However, this patient was hospitalised a further four times for AHRF during the follow-up year and required NIV and endotracheal ventilation.

A total of $50 \%$ of the study patients were hospitalised for AHRF at least on one occasion during the 1-yr follow-up period. The proportions of patients with or without LTD-NIV were similar, as was the proportion of patients with or without COPD (table 4). There was a high requirement for endotracheal intubation in COPD patients with LTD-NIV (60\%).

The 1-yr mortality figures were similar in the non-COPD patients whether or not these patients had LTD-NIV, and were similar to the mortality rate in COPD patients without LTDNIV (table 4). Most of the deaths in the non-COPD subgroup were in the oldest patients. The mortality rate in COPD
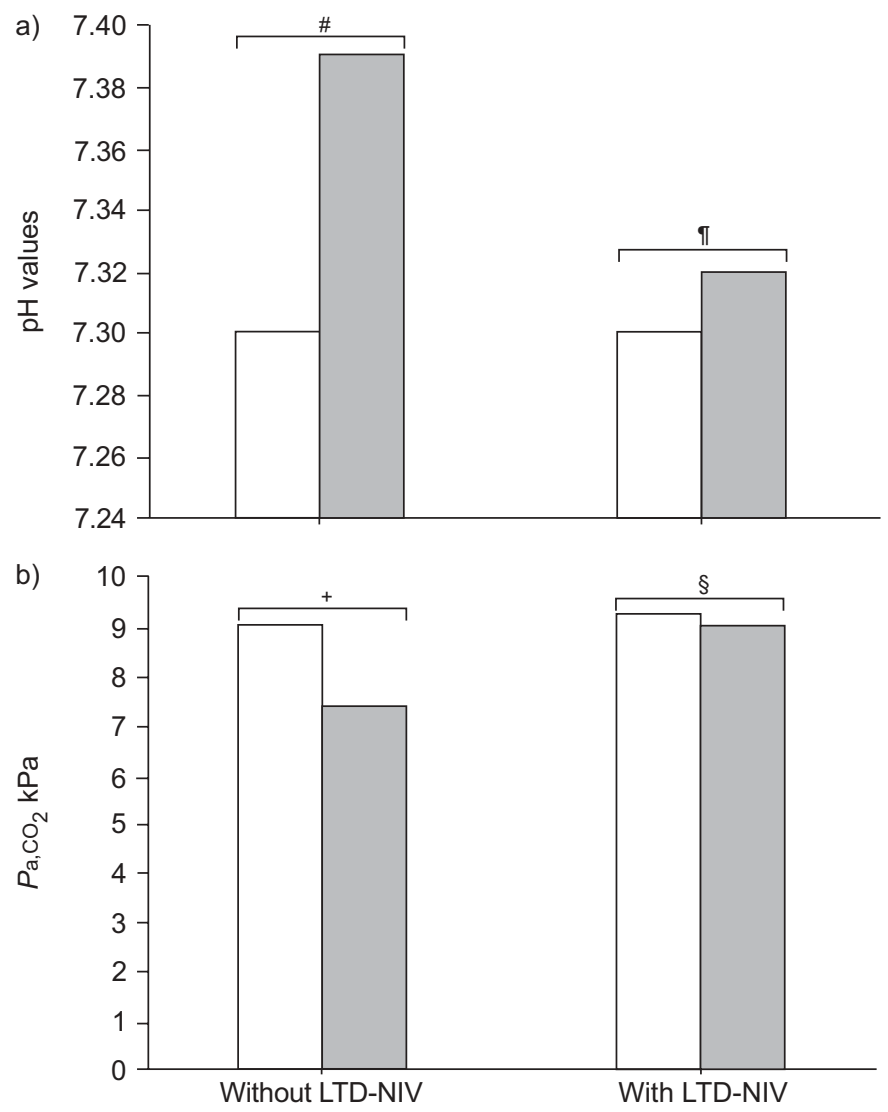

FIGURE 2. Assessments of a) arterial pH and b) carbon dioxide arterial tension $\left(\mathrm{Pa}, \mathrm{CO}_{2}\right)$ values on spontaneous breathing in patients with $(\mathrm{n}=31)$ and without ( $n=69)$ long-term dependency on noninvasive ventilation (LTD-NIV) during the first $24 \mathrm{~h}$ after admission into the respiratory intermediate care unit. Data values presented are those obtained at admission $(\square)$ and after $24 \mathrm{~h}$ of NIV ( $\square$ ). LTD-NIV was associated with a significantly less correction of $\mathrm{pH}$ and $\mathrm{Pa}, \mathrm{CO}_{2}$ levels measured $24 \mathrm{~h}$ after admission. All paired comparisons were performed with Wilcoxon's signed-rank test. ${ }^{*}: p<0.0001 ;{ }^{\bullet}: p=0.74 ;^{+}: p=0.0001 ;{ }^{\S}: p=0.41$.

patients with LTD-NIV was high, although the number of patients was too small to reach statistical significance.

\section{DISCUSSION}

The current study shows that $31 \%$ of patients admitted to the authors' RICU with AHRF and without previous home ventilation had LTD-NIV on discharge. Furthermore, the incidence of LTD-NIV is twice as frequent in non-COPD patients $(39 \%)$ than in COPD patients $(19 \%)$. These proportions illustrate the significance of ventilatory dependency in post-acute care and are consistent with the recent development of RICUs [19-21]. RICUs are appropriate structures for assessing this phenomenon because of the extensive NIV use in these units and the higher proportion of patients admitted having chronic respiratory failure and, therefore, more prone to ventilatory dependency.

Few studies have addressed the issue of LTD-NIV after an episode of AHRF and have reported a lower proportion of such patients. This is probably due to differences in the recruitment of patients or in the place where episodes of AHRF 

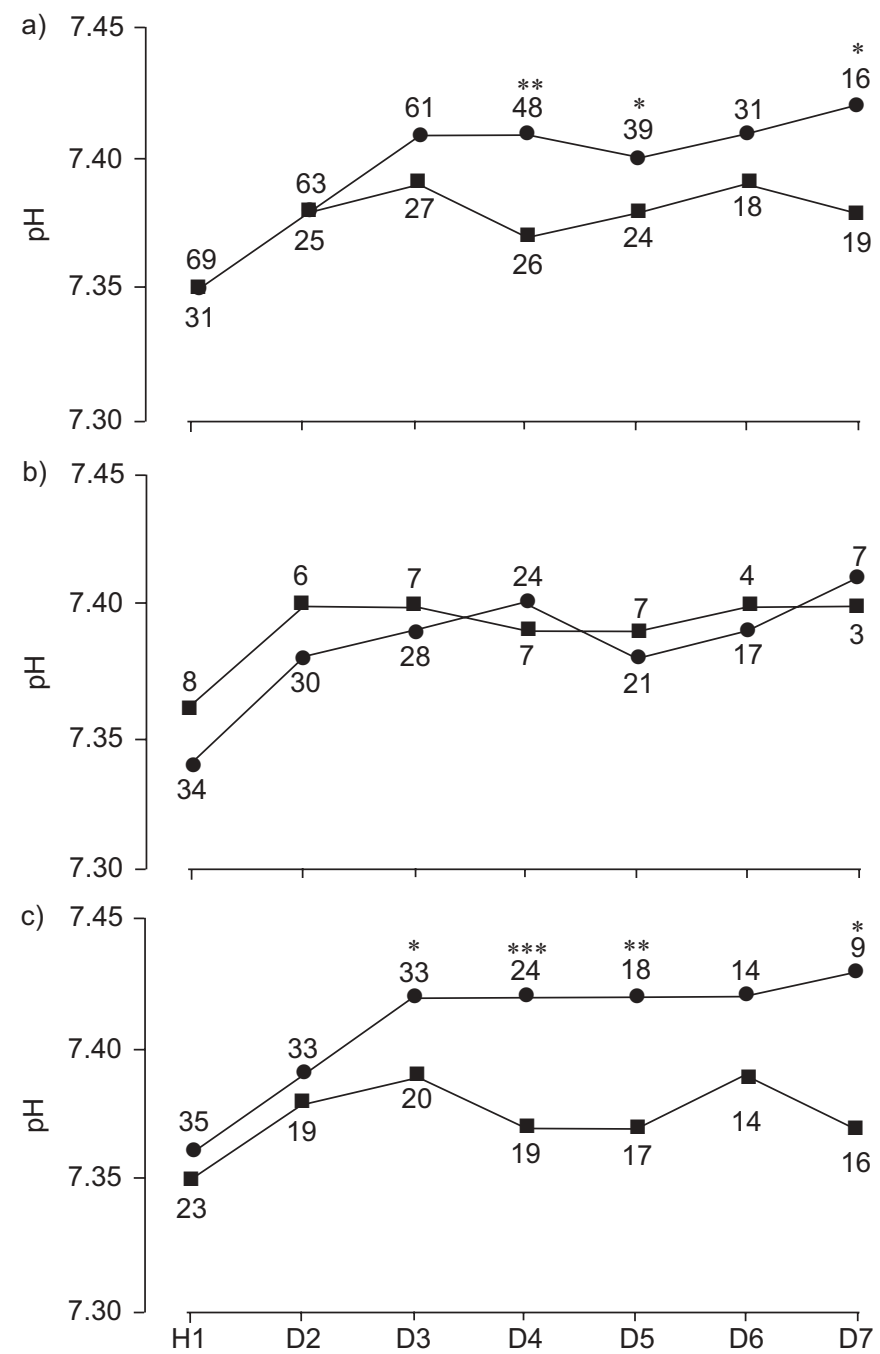

FIGURE 3. Change in arterial pH over time in a) all patients, b) chronic obstructive pulmonary disease (COPD) patients and c) non-COPD patients when measured under mechanical ventilation in the respiratory intermediate care unit. Change in arterial pH over 7 days significantly differed (as assessed using two-way ANOVA) between patients with $(\bullet)$ and without (-) subsequent long-term dependency on noninvasive ventilation (LTD-NIV), but only in the non-COPD subgroup ( $p=0.0098$ ), with day-by-day significant differences on days $3,4,5$ and 7 (as assessed by the Mann-Whitney U-test). Numbers of patients are mentioned at all time points. ${ }^{*}: p<0.05,{ }^{* *}: p<0.01,{ }^{* *}: p<0.001$.

are managed: $3-6 \%$ of adult patients after admission to ICU have been reported to require prolonged mechanical ventilation [22-24], but these data relate mainly to tracheostomised patients managed in the ICU. A recent Italian survey of 26 RICUs [20], with similar organisation compared with that of the present study, reported $22 \%$ of LTD-NIV in a population including post-surgical patients $(8 \%)$ and/or patients with hypoxaemic respiratory failure $(24 \%)$. This population included fewer patients with chronic respiratory failure and explains the lower rate of LTD-NIV, compared with patients in the current study. As only limited data are available in the literature, the aim was to identify predictive factors of LTDNIV in patients discharged from RICU after an episode of
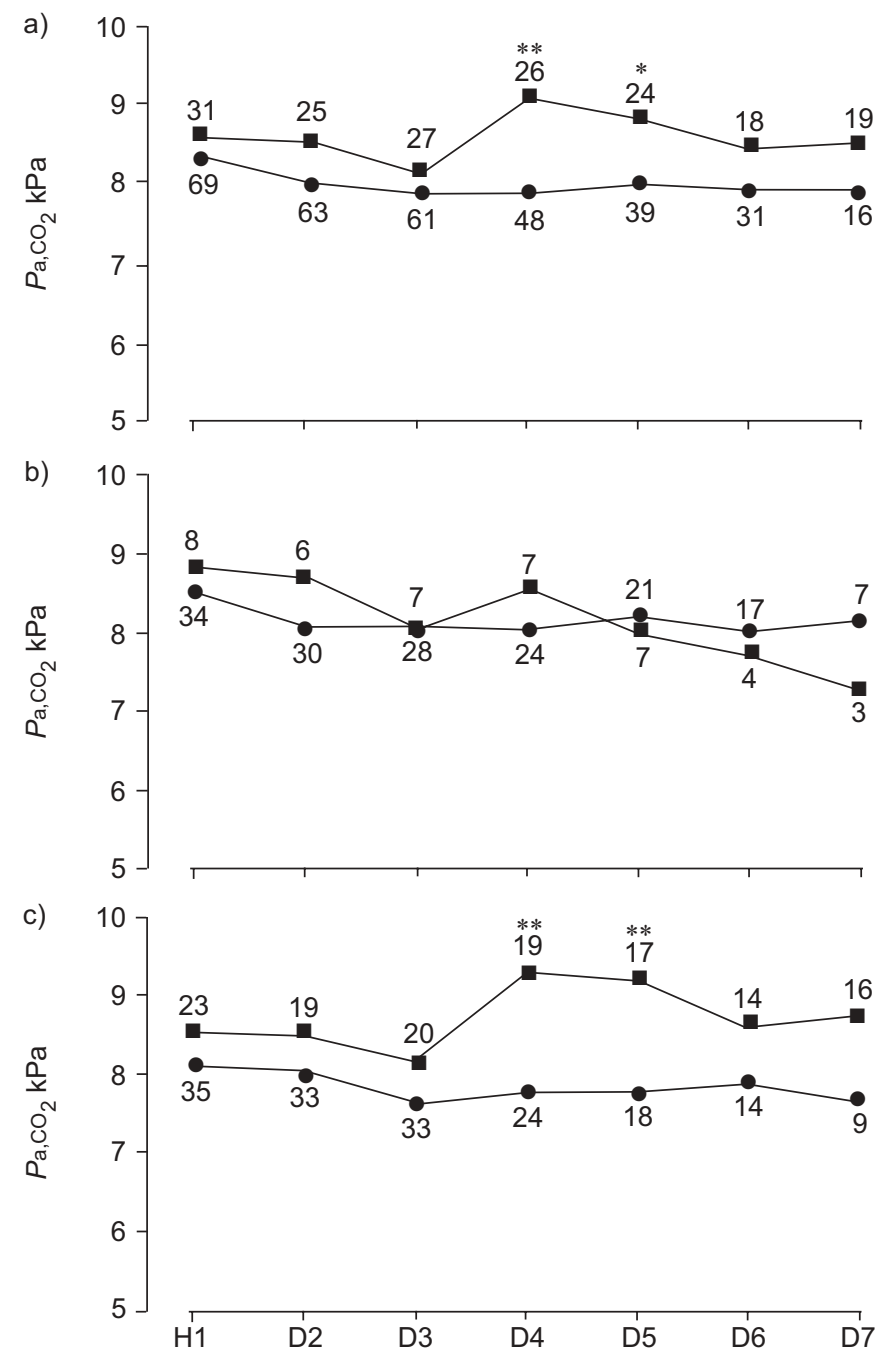

FIGURE 4. Change in carbon dioxide arterial tension $\left(\mathrm{Pa}_{1} \mathrm{CO}_{2}\right)$ over time in a) all patients, b) chronic obstructive pulmonary disease (COPD) patients and c) nonCOPD patients when measured under mechanical ventilation in the respiratory intermediate care unit. Change in arterial $\mathrm{Pa}_{2} \mathrm{CO}_{2}$ over 7 days significantly differed (as assessed using two-way ANOVA) between patients with $(\bullet)$ and without $(\mathbf{\square})$ subsequent long-term dependency on noninvasive ventilation in the whole sample $(p=0.02)$ and in the non-COPD subgroup $(p=0.021)$ with significant interaction with time ( $p=0.006$ and $p=0.025$, respectively) and with day-by-day significant differences on days 4 and 5 (as assessed from Mann-Whitney U-test). Numbers of patients are mentioned at all time points. ${ }^{*}: p<0.05,{ }^{* *}: p<0.01$.

AHRF; first, at stable state during the 6 months prior to the episode of AHRF and, secondly, at admission to the RICU. Finally, the outcome in patients with and without LTD-NIV during a 1-yr follow-up after discharge from the RICU was compared.

Among parameters at stable state, the present study demonstrates that the severity of the underlying chronic respiratory failure (attested by a lower $\mathrm{pH}$ during a 6-month period before the AHRF episode and not by lung function test parameters) is a strong predictive factor for LTD-NIV on discharge after an episode of AHRF. The occurrence of LTD-NIV also varies depending on the cause of chronic respiratory failure, as a $\mathrm{pH}$ 


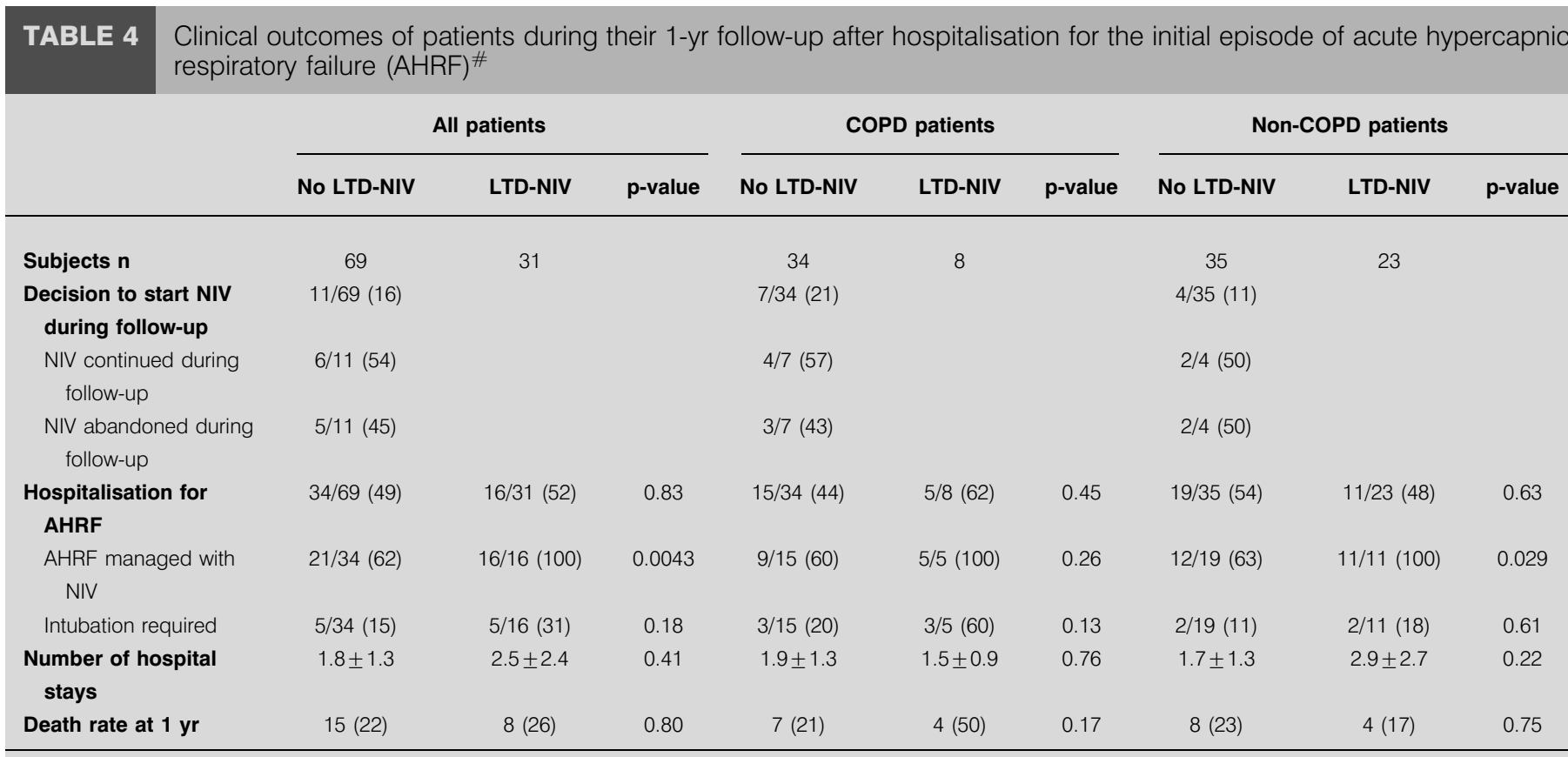

Data are presented as $\mathrm{n} / \mathrm{n}(\%)$ or mean $\pm \mathrm{SD}$, unless otherwise indicated. COPD: chronic obstructive pulmonary disease. \#: Long-term dependency on noninvasive ventilation (LTD-NIV) was defined by the same criteria as were used during the initial episode of AHRF on inclusion.

value $\leqslant 7.38$ at baseline was associated with a 3-fold-higher probability of LTD-NIV on discharge in non-COPD patients compared to patients with COPD. Previous studies showed that the severity of $\mathrm{ABG}$ impairment at basal state is an independent predictor of hospitalisation for acute exacerbation in patients with moderate-to-severe COPD [25] and influences long-term mortality after hospital discharge [26]. The presented results add further information to these studies by showing that this parameter is also an independent predictor of LTD-NIV after an AHRF episode in the RICU. However, this information is not always available at admission.

Among parameters collected at admission into the RICU, the authors found that patient severity (based on ABGs and SAPS score) does not predict LTD-NIV on discharge. Furthermore, the rates of $\mathrm{pH}$ and $\mathrm{Pa}_{1} \mathrm{CO}_{2}$ improvement on spontaneous breathing are early predictors for LTD-NIV. The current results are different from previous studies which have described predictive factors of immediate NIV success/failure, but have mostly considered patients with COPD and have used intubation and/or mortality as primary endpoints [1, 27-31]. These studies did not provide any information about LTD-NIV on discharge. The present results suggest that: 1) patient severity at admission cannot be used to assess a possible occurrence of LTD-NIV on discharge; and 2) successful management of an episode of AHRF under NIV, either in COPD or non-COPD patients, does not exclude the potential need for long-term NIV.

Concerning the outcome, the COPD patients with LTD-NIV had a high 1-yr death rate without any fall in their hospitalisation rate. These COPD patients may have been nonresponders to domiciliary NIV, but the fact that $75 \%$ of them had been hospitalised at least once for AHRF during the three previous years (with a 50\% intubation rate) also suggests that they had advanced COPD disease, less likely to be influenced by domiciliary NIV. The presented results are consistent with published findings showing a poor outcome in COPD patients after successful treatment for AHRF [32] and no benefit in survival when long-term NIV is started in COPD $[11,12,33]$. Future studies are needed to address the impact of long-term NIV on other important outcomes, such as hospitalisation rates [14] and health status; this latter parameter was not assessed in the current study because of its retrospective design. This study, therefore, suggests that LTD-NIV after an episode of AHRF is a marker of disease severity during COPD. This does not apply to non-COPD patients, probably because these patients are suffering from a range of different pulmonary disorders.

The main limitations of this study stem from its retrospective design and the fact that the present authors used a procedure to stop NIV that has not been validated previously. To the best of the present authors' knowledge, there are no guidelines or prospectively standardised procedures to perform weaning from NIV, as compared with published weaning protocols for invasive ventilation. Although the patients in this study were managed using standardised procedures throughout the study period, by an experienced medical and nursing team, the consistent practice in the RICU does not replace a prospectively validated weaning protocol and a prospectively validated definition of LTD-NIV.

In conclusion, the current study shows that long-term dependency on noninvasive ventilation after acute hypercapnic respiratory failure is a common situation in respiratory intermediate care units, especially in patients with nonCOPD causes of respiratory failure. Prediction of long-term 
dependency on noninvasive ventilation in the respiratory intermediate care unit is based on the severity of the underlying respiratory impairment at stable state ( $\mathrm{pH}$ level), but not on severity parameters on admission to the respiratory intermediate care unit, such as arterial blood gases or simplified acute physiology score. Outcome remains poor, especially in chronic obstructive pulmonary disease patients. The approach of the present authors constitutes the first way to delineate the issue of long-term dependency on noninvasive ventilation in the respiratory intermediate care unit and raises the need for prospective validation of a weaning protocol in patients managed by noninvasive ventilation for an acute hypercapnic respiratory failure episode.

\section{ACKNOWLEDGEMENTS}

The authors are grateful to R. Medeiros (Medical Editor, Rouen University Hospital, Rouen, France) for his valuable contribution in editing the manuscript and to F. Dugardin and D. Métayer (L'ANTADIR, Paris, France) for their technical help.

\section{REFERENCES}

1 Brochard L, Mancebo J, Wysocki M, et al. Noninvasive ventilation for acute exacerbations of chronic obstructive pulmonary disease. N Engl J Med 1995; 333: 817-822.

2 Plant PK, Owen JL, Elliott MW. Early use of non-invasive ventilation for acute exacerbations of chronic obstructive pulmonary disease on general respiratory wards: a multicentre randomised controlled trial. Lancet 2000; 355: 1931-1935.

3 Leger P, Bedicam JM, Cornette A, et al. Nasal intermittent positive pressure ventilation. Long-term follow-up in patients with severe chronic respiratory insufficiency. Chest 1994; 105: 100-105.

4 Simonds AK, Elliott MW. Outcome of domiciliary nasal intermittent positive pressure ventilation in restrictive and obstructive disorders. Thorax 1995; 50: 604-609.

5 Mehta S, Hill NS. Noninvasive ventilation. Am J Respir Crit Care Med 2001; 163: 540-577.

6 Gay PC, Hubmayr RD, Stroetz RW. Efficacy of nocturnal nasal ventilation in stable, severe chronic obstructive pulmonary disease during a 3-month controlled trial. Mayo Clinic Proc 1996; 71: 533-542.

7 Criner GJ, Brennan K, Travaline JM, Kreimer D. Efficacy and compliance with noninvasive positive pressure ventilation in patients with chronic respiratory failure. Chest 1999; 116: 667-675.

8 Make BJ, Hill NS, Goldberg AI, et al. Mechanical ventilation beyond the intensive care unit. Report of a consensus conference of the American College of Chest Physicians. Chest 1998; 113: Suppl. 5, 289S-344S.

9 Clinical indications for noninvasive positive pressure ventilation in chronic respiratory failure due to restrictive lung disease, COPD, and nocturnal hypoventilation. A consensus conference report. Chest 1999; 116: 521-534.

10 Hill NS. Noninvasive ventilation in chronic obstructive pulmonary disease. Clin Chest Med 2000; 21: 783-797.
11 Clini E, Sturani C, Rossi A, et al. The Italian multicentre study on noninvasive ventilation in chronic obstructive pulmonary disease patients. Eur Respir J 2002; 20: 529-538.

12 Muir JF, De la Salmonière P, Cuvelier A, Chevret S, Cilt C, Chastang C. Home NIPPV + oxygen versus long-term oxygen therapy alone in severe hypercapnic COPD patients: a European multicentre study. Am J Respir Crit Care Med 2000; 161: A262.

13 Cuvelier A, Muir JF. Noninvasive ventilation and obstructive lung diseases. Eur Respir J 2001; 17: 1271-1281.

14 Tuggey JM, Plant PK, Elliott MW. Domiciliary noninvasive ventilation for recurrent acidotic exacerbations of COPD: an economic analysis. Thorax 2003; 58: 867-871.

15 Lightowler JV, Wedzicha JA, Elliott MW, Ram FS. Noninvasive positive pressure ventilation to treat respiratory failure resulting from exacerbations of chronic obstructive pulmonary disease: Cochrane systematic review and metaanalysis. BMJ 2003; 326: 185.

16 Keenan SP, Sinuff T, Cook DJ, Hill NS. Which patients with acute exacerbation of chronic obstructive pulmonary disease benefit from noninvasive positive-pressure ventilation? A systematic review of the literature. Ann Intern Med 2003; 138: 861-870.

17 Robino C, Faisy C, Diehl JL, Rezgui N, Labrousse J, Guerot E. Effectiveness of non-invasive positive pressure ventilation differs between decompensated chronic restrictive and obstructive pulmonary disease patients. Intensive Care Med 2003; 29: 603-610.

18 American Thoracic Society. International consensus conferences in intensive care medicine: noninvasive positive pressure ventilation in acute respiratory failure. Am J Respir Crit Care Med 2001; 163: 283-291.

19 Nava S, Confalonieri M, Rampulla C. Intermediate respiratory intensive care units in Europe: a European perspective. Thorax 1998; 53: 798-802.

20 Confalonieri M, Gorini M, Ambrosino N, Mollica C, Corrado A. Respiratory intensive care units in Italy: a national census and prospective cohort study. Thorax 2001; 56: 373-378.

21 Corrado A, Roussos C, Ambrosino N, et al. Respiratory intermediate care units: a European survey. Eur Respir J 2002; 20: 1343-1350.

22 Daly BJ, Rudy EB, Thompson KS, Happ MB. Development of a special care unit for chronically critically ill patients. Heart Lung 1991; 20: 45-51.

23 Stauffer JL, Fayter NA, Graves B, Cromb M, Lynch JC, Goebel P. Survival following mechanical ventilation for acute respiratory failure in adult men. Chest 1993; 104: 1222-1229.

24 Carson SS, Bach PB, Brzozowski L, Leff A. Outcomes after long-term acute care. An analysis of 133 mechanically ventilated patients. Am J Respir Crit Care Med 1999; 159: 1568-1573.

25 Kessler R, Faller M, Fourgaut G, Mennecier B, Weitzenblum E. Predictive factors of hospitalization for acute exacerbation in a series of 64 patients with chronic obstructive pulmonary disease. Am J Respir Crit Care Med 1999; 159: 158-164.

26 Seneff MG, Wagner DP, Wagner RP, Zimmerman JE, Knaus WA. Hospital and 1-year survival of patients admitted to intensive care units with acute exacerbation 
of chronic obstructive pulmonary disease. JAMA 1995; 274: 1852-1857.

27 Soo Hoo GW, Santiago S, Williams AJ. Nasal mechanical ventilation for hypercapnic respiratory failure in chronic obstructive pulmonary disease: determinants of success and failure. Crit Care Med 1994; 22: 1253-1261.

28 Ambrosino N, Foglio K, Rubini F, Clini E, Nava S, Vitacca M. Non-invasive mechanical ventilation in acute respiratory failure due to chronic obstructive pulmonary disease: correlates for success. Thorax 1995; 50: 755-757.

29 Putinati S, Ballerin L, Piattella M, Panella GL, Potena A. Is it possible to predict the success of non-invasive positive pressure ventilation in acute respiratory failure due to COPD? Respir Med 2000; 94: 997-1001.
30 Anton A, Guell R, Gomez J, et al. Predicting the result of noninvasive ventilation in severe acute exacerbations of patients with chronic airflow limitation. Chest 2000; 117: 828-833.

31 Antonelli M, Conti G, Moro ML, et al. Predictors of failure of noninvasive positive pressure ventilation in patients with acute hypoxemic respiratory failure: a multi-center study. Intensive Care Med 2001; 27: 1718-1728.

32 Breen D, Churches T, Hawker F, Torzillo PJ. Acute respiratory failure secondary to chronic obstructive pulmonary disease treated in the intensive care unit: a long term follow up study. Thorax 2002; 57: 29-33.

33 Casanova C, Celli BR, Tost L, et al. Long-term controlled trial of nocturnal nasal positive pressure ventilation in patients with severe COPD. Chest 2000; 118: 1582-1590. 